\title{
Delayed Birth-Related Femur Fracture after Cesarean Section: A Case Report
}

Yuji Kanai, MD ${ }^{1,2}$ Yoshinobu Honda, MD, PhD ${ }^{1}$ Tsuyoshi Honda, MD, PhD 3 Minoru Sanpei, MD, $\mathrm{PhD}^{3}$ ${ }^{1}$ Department of Premature and Newborn, Iwaki Kyouritsu Hospital,
Iwaki, Japan
${ }^{2}$ Department of Pediatrics, Fukushima Medical University School of
Medicine, Fukushima Medical University, Fukushima, Japan
${ }^{3}$ Department of Obstetrics and Gynecology, Iwaki Kyouritsu Hospital,
Iwaki, Japan

Am J Perinatol Rep 2018;8:e158-e160.
Address for correspondence Yuji Kanai, MD, Department of Pediatrics, Fukushima Medical University, 1, Hikarigaoka, Fukushima City, Fukushima 960-1295, Japan (e-mail: kebin@fmu.ac.jp).

\begin{abstract}
Background Birth-related femur fractures are rare. They have been reported following difficult delivery and are commonly diagnosed immediately after birth or on the following day. However, some birth-related femur fractures are diagnosed several days after birth. Mechanisms underlying the development of delayed femur fracture are incompletely understood.

Case We report the case of a girl diagnosed with a left spiral femur fracture with associated edema and hypoesthesia on postpartum day 9. A nondisplacement femur fracture was identified on plain radiography at birth after cesarean section; however, it was undiagnosed due to the lack of symptoms. She was treated with plaster cast fixation; the physical examination at age 1 was normal. Herein, the diagnosis of birthrelated femur fracture according to plain radiography findings suggests that the symptoms related to birth trauma develop several days after birth.

\section{Keywords}

- femur fracture

- cesarean section

- breech delivery

- delayed diagnosis

Conclusion Birth-related fracture should be considered in all neonatal femur fracture cases and in patients with no symptoms at birth. Neonates delivered in the breech presentation by cesarean section should be carefully monitored after birth. The findings of the present case further our understanding of the pathogenic mechanisms underlying delayed diagnosis of birth-related femur fracture.
\end{abstract}

Cesarean section (CS) is a common delivery method for breech birth to prevent trauma. ${ }^{1}$ Birth trauma associated with CS rarely causes femur fracture. ${ }^{2}$ Femur fracture incidence was reportedly 0.077 to 0.13 per 1,000 deliveries. ${ }^{3,4}$ Mean time for diagnosis was reportedly $6.3^{3}$ or 4 days ${ }^{2}$ with some cases diagnosed several days after birth. However, most femur fracture cases were reportedly diagnosed on the day of birth or the following day. ${ }^{3,5,6}$ Birth-related femur fracture may be diagnosed almost immediately after birth in cases of difficult extractions, wherein abnormal cracking sounds are heard at birth; however, some cases are diag- nosed several days after birth ${ }^{3}$; the reasons for this have not yet been understood.

Herein, we present a neonatal case of nondisplacement femur fracture at birth and spiral femur fracture diagnosed on postpartum day 9 after CS for breech delivery without a difficult extraction or abnormal sounds noted at birth.

\section{Case Report}

A healthy 27-year-old pregnant female was admitted for planned CS for breech presentation at 38-week gestational received

March 8, 2018

accepted after revision

June 14, 2018
DOI https://doi.org/

$10.1055 / \mathrm{s}-0038-1667190$ ISSN 2157-6998.
Copyright $\odot 2018$ by Thieme Medical Publishers, Inc., 333 Seventh Avenue, New York, NY 10001, USA. Tel: +1(212) 584-4662.
License terms

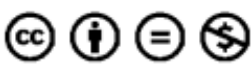



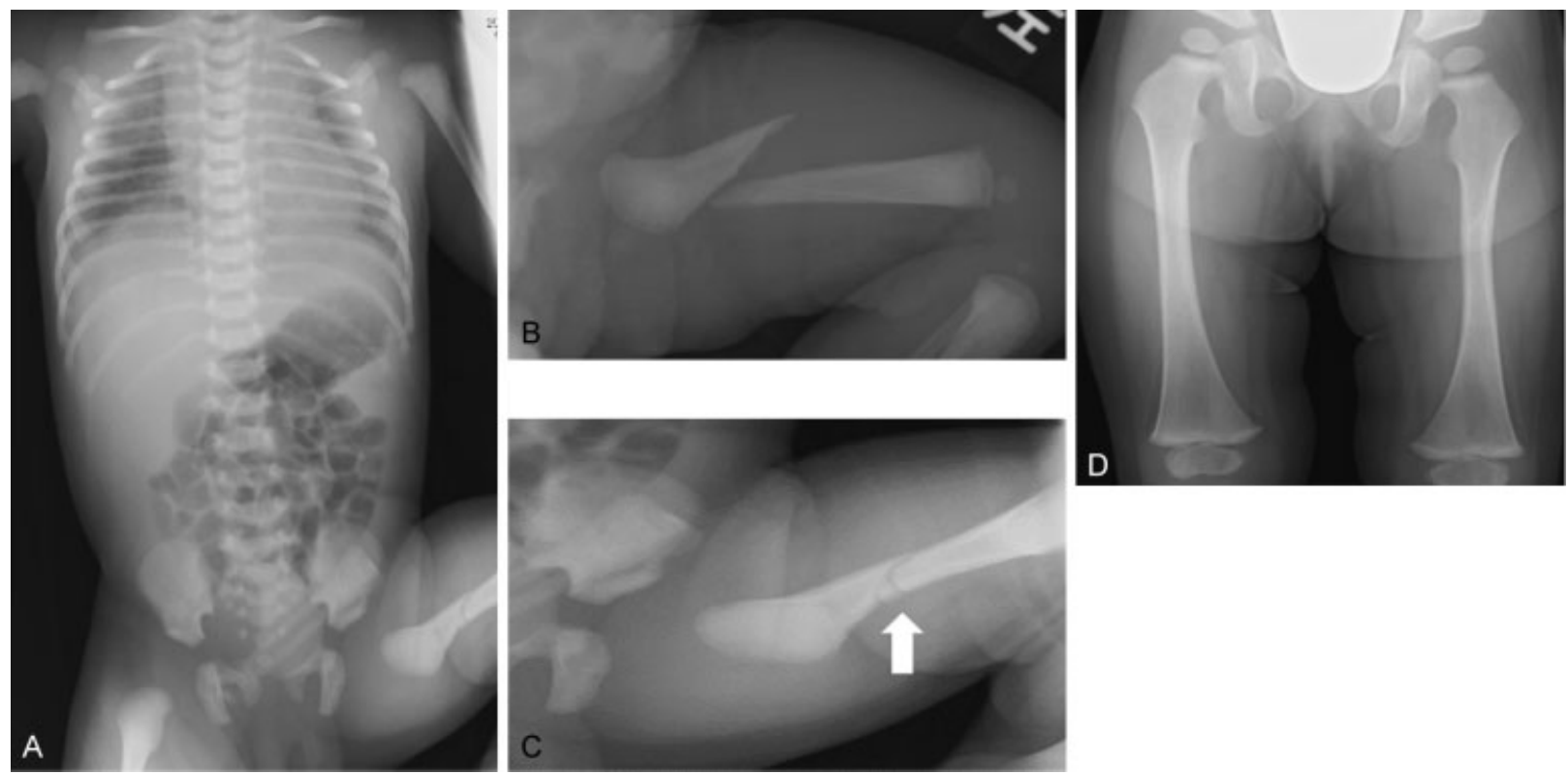

Fig. 1 Radiography findings of the patient. (A) Whole body plain radiography at birth. (B) Magnified plain radiography of the left femur on postpartum day 9 demonstrating spiral fracture. (C) Magnified plain radiography of the left femur at birth demonstrating a nondisplacement fracture (white arrow). (D) Plain radiography of both femurs at age 1 year.

age. Her pregnancy had normal clinical course. A neonatologist was present at the time of childbirth. The neonate weighted 2,930 g with Appearance, Pulse, Grimace, Activity, and Respiration scores of 8 and 9 at 1 and 5 minutes, respectively, and with no difficultly in extraction or abnormal cracking sounds noted by the obstetrician and neonatologist at birth. As she experienced an episode of $\mathrm{O}_{2}$ desaturation with tachypnea 2 hours after birth, whole body plain radiography was performed and prominent bilateral pulmonary blood vessel shadowing was demonstrated (-Fig. 1A). Two pediatricians confirmed the plain radiography findings. Laboratory analyses, including serum levels of calcium, phosphorus, and alkaline phosphatase, had normal outcomes. Transient tachypnea of the newborn was diagnosed; she was admitted to our neonatal intensive care unit (NICU). Nasal continuous positive airway pressure was initiated; her respiratory failure was improved within 24 hours. On day 3, she was moved from the NICU to rooming-in. From day 5, her mother reported loud crying during each diaper change. She was discharged on postpartum day 8. On postpartum day 9 , her mother noticed no movement of the patient's left leg and edema over the left femur. She, therefore, consulted our pediatric clinic the following day. Initial examination revealed erythema and edema over the left leg; radiographic examination revealed spiral femur fracture (-Fig. 1B). We did not observe other bone fractures, wounds, muscular hypotonia, healing impairment, or blue sclera. Reconsideration of the plain radiography performed at birth revealed a left nondisplacement femur fracture (-Fig. 1C). The possibility of abuse or an underlying disease like congenital osteoplasia was considered low; consequently, we diagnosed birth-related femur fracture. The fracture was treated with plaster cast fixation.
Plain radiography on day 21 posttreatment revealed callus formation; subsequently, the cast was removed. After 2 months, the fracture had completely healed. At 1 year, there was no evidence of transformation, difference in length of femur (-Fig. 1D), or abnormality in walking. We repeatedly explained to the mother that the baby got broken due to birth trauma. Moreover, she did not experience any trauma as a result of the newborn's injuries. Written informed consent was obtained from the patient for publication of this case presentation and any accompanying images.

\section{Discussion}

We observed a nondisplacement femur fracture at birth by plain radiography that developed into spiral fracture. To the best of our knowledge, this is the first case that birth-related femur fracture may present several days after birth.

For births with breech presentation, CS is recommended, ${ }^{1}$ which increases the risk of femur fracture. ${ }^{2}$ Most birthrelated femur fractures are diagnosed early because of difficult extraction or abnormal cracking sounds noted at birth. However, the lack of symptoms delays the diagnosis of some birth-related femur fractures. Therefore, they can be categorized according to the day of diagnosis: those diagnosed the following day and those diagnosed on or after postpartum day 3. Symptoms such as soft-tissue swelling, joint stiffness, focal tenderness, and irritability are late findings and may explain the delayed diagnosis. ${ }^{7}$ However, the mechanisms and clinical course of such fractures currently are incompletely understood. Difficult extraction and abnormal sounds at delivery were not observed in our case; however, nondisplacement femur fracture at birth possibly developed into spiral fracture, which was diagnosed when 
overt symptoms appeared. To the best of our knowledge, this is the first reported case of "delayed" birth-related femur fracture that became apparent several days after birth.

Herein, nondisplacement femur fracture at birth developed into spiral fracture over several days, suggesting that the fracture developed as a result of the subsequent application of an external force to this bone region. Radiographic propagation of spiral fractures and six simple principles were reportedly caused by torsional force in rabbits. ${ }^{8}$ Our case involved a nondisplacement femur fracture at birth, which was considered to be caused by the dominant torsion at the time of delivery despite no difficulty in extraction. The fracture was not immediately diagnosed because the nondisplacement femur fracture was slight and there were no overt symptoms until a few days after birth. Her mother noted repeated episodes of excessive crying during diaper changes. Repeated torsion involved in lifting both legs during diaper change may have caused the progression to spiral fracture. An early treatment of the nondisplacement fracture can prevent this.

Birth-related injury, systemic bone disease, and abuse are recognized causes of femur fracture during the neonatal period. ${ }^{9}$ Femur fracture incidence due to abuse in infants aged less than 12 months was reported to be 16.7 to $35.2 \%$, and spiral fractures are reportedly not associated with abuse. $^{9}$ Therefore, all femur fractures of unknown origin observed in infants before the development of the ability to walk should be completely investigated. Age, suspicious history, and additional injuries may indicate abuse. ${ }^{9,10}$ Our patient was diagnosed with birth-related femur fracture using plain radiography findings at birth. However, in the absence of imaging, abuse would have been considered. Plain radiography has a utility in identifying bone fractures; however, there is a lack of evidence demonstrating the utility of performing plain radiography in all neonates delivered by CS in the breech presentation. The method of delivery should be confirmed in all femur fracture cases presenting several days after birth.

To the best of our knowledge, no studies have reported this rare radiographic finding just after birth. We believe our findings provide novel information regarding the natural history of birth injury.

Femur fractures may develop during CS for breech delivery; therefore, infants who are delivered using this method should be carefully evaluated.

\section{Authors' Contributions}

Y.K. managed the patient and was responsible for the concept, design, and writing of the final version of the manuscript. Y.H. managed the patient and made substantial contributions in drafting and revising the manuscript. T.H. and M.S. participated in the management of the patient and revised the manuscript. All authors have read and approved the final manuscript.

\section{Funding}

None.

\section{Acknowledgment}

The authors would like to thank Enago (www.enago.jp) for the English language review.

\section{References}

1 ACOG Committee on Obstetric Practice. ACOG Committee Opinion No. 340. Mode of term singleton breech delivery. Obstet Gynecol 2006;108(01):235-237

2 Kancherla R, Sankineani SR, Naranje S, et al. Birth-related femoral fracture in newborns: risk factors and management. J Child Orthop 2012;6(03):177-180

3 Morris S, Cassidy N, Stephens M, McCormack D, McManus F. Birth-associated femoral fractures: incidence and outcome. J Pediatr Orthop 2002;22(01):27-30

4 Toker A, Perry ZH, Cohen E, Krymko H. Cesarean section and the risk of fractured femur. Isr Med Assoc J 2009;11(07):416-418

5 Basha A, Amarin Z, Abu-Hassan F. Birth-associated long-bone fractures. Int J Gynaecol Obstet 2013;123(02):127-130

6 Matsubara S, Izumi A, Nagai T, Kikkawa I, Suzuki M. Femur fracture during abdominal breech delivery. Arch Gynecol Obstet 2008;278(02):195-197

7 Cebesoy FB, Cebesoy O, Incebiyik A. Bilateral femur fracture in a newborn: an extreme complication of cesarean delivery. Arch Gynecol Obstet 2009;279(01):73-74

8 O'Connor-Read L, Teh J, Willett K. Radiographic evidence to help predict the mechanism of injury of pediatric spiral fractures in nonaccidental injury. J Pediatr Orthop 2007;27(07):754-757

9 Wood JN, Fakeye O, Mondestin V, Rubin DM, Localio R Feudtner C. Prevalence of abuse among young children with femur fractures: a systematic review. BMC Pediatr 2014 $14: 169$

10 Baldwin K, Pandya NK, Wolfgruber H, Drummond DS, Hosalkar HS. Femur fractures in the pediatric population: abuse or accidental trauma? Clin Orthop Relat Res 2011;469(03):798-804 